\title{
First-principles study on the stability and electronic structure of monolayer GaSe with trigonal-antiprismatic structure
}

\author{
Hirokazu Nitta, ${ }^{1}$ Takahiro Yonezawa $\odot,{ }^{1}$ Antoine Fleurence $\odot,{ }^{1}$ Yukiko Yamada-Takamura, ${ }^{1}$ and Taisuke Ozaki ${ }^{2}$ \\ ${ }^{1}$ School of Materials Science, Japan Advanced Institute of Science and Technology (JAIST), 1-1 Asahidai, Nomi, Ishikawa 923-1292, Japan \\ ${ }^{2}$ Institute for Solid State Physics, The University of Tokyo, 5-1-5 Kashiwanoha, Kashiwa, Chiba 277-8581, Japan
}

(Received 4 June 2020; revised 22 October 2020; accepted 26 October 2020; published 4 December 2020)

\begin{abstract}
The structural stability and electronic states of GaSe monolayer with trigonal-antiprismatic (AP) structure, which is a recently discovered polymorph, were studied by first-principles calculations. The AP-phase GaSe monolayer was found stable, and the differences in energy and lattice constant were small when compared to those calculated for a GaSe monolayer with conventional trigonal-prismatic $(\mathrm{P})$ structure which was found to be the ground state. Moreover, it was revealed that the relative stability of P-phase and AP-phase GaSe monolayers reverses under tensile strain. These calculation results provide insight into the formation mechanism of AP-phase GaSe monolayers in epitaxially grown GaSe thin films.
\end{abstract}

DOI: 10.1103/PhysRevB.102.235407

\section{INTRODUCTION}

Two-dimensional materials exhibit many unique physical properties compared to bulk materials. In recent years, the study of layered metal-chalcogenides (LMCs) has been a topic of high interest because they exhibit a wide variety of properties depending on composition and number of layers [1,2]. The bonding between the atoms in a monolayer of LMCs is of covalent and/or ionic type, while the bonding between the layers is of the molecular, van der Waals type.

Gallium selenide $(\mathrm{GaSe})$ is a LMC with a $2-\mathrm{eV}$ band gap, and is known to be a good nonlinear optical crystal owing to its noncentrosymmetric crystal structure [3]. A monolayer of GaSe is composed of covalently bonded quadruple atomic layers in a Se-Ga-Ga-Se sequence, as illustrated in Fig. 1(a). This is for the trigonal-prismatic GaSe structure. We name this GaSe crystal, which adopts a conventional, wurtzitelike structure, the "prismatic $(\mathrm{P})$ phase," because Se atoms are coordinated in the form of a triangular prism with respect to the Ga dimer as shown in Fig. 1(a). Bulk GaSe with monolayers stacked vertically via van der Waals forces crystallizes in several polytypes with different stacking sequences: $\beta$-GaSe, $\varepsilon$-GaSe, $\gamma$-GaSe, and $\delta$-GaSe $[3,4]$. The most commonly found polytypes are $\varepsilon$ - and $\gamma$-GaSe [5]. The band gap of $\varepsilon-\mathrm{GaSe}$ is theoretically predicted to increase from $1.06 \mathrm{eV}$ (bulk) to $2.25 \mathrm{eV}$ (monolayer) by decreasing the number of layers [6], and this tendency has been confirmed experimentally by scanning tunneling spectroscopy (STS) [7] and cathodoluminescence (CL) [8], where the gap of GaSe monolayer was measured to be $3.5 \pm 0.05 \mathrm{eV}$ and $3.3 \mathrm{eV}$,

Published by the American Physical Society under the terms of the Creative Commons Attribution 4.0 International license. Further distribution of this work must maintain attribution to the author(s) and the published article's title, journal citation, and DOI. respectively. Recently, GaSe has been predicted to possess promising properties: $\varepsilon$-GaSe is expected to turn into a threedimensional (3D) topological insulator when a tensile strain of $3 \%$ or more is applied [9], while a hole-doped monolayer shows tunable ferromagnetism and half-metallicity [10]. Furthermore, a spin-orbit coupling (SOC) ten times stronger than that for GaAs was observed experimentally for electron-doped $\varepsilon$-GaSe thin flakes with 10 and $25 \mathrm{~nm}$ thicknesses [11].

We have recently succeeded in growing epitaxial $\mathrm{GaSe}(0001)$ thin films on $\mathrm{Ge}(111)$ substrates using molecular beams of Ga and Se [12] through van der Waals epitaxy [13]. In a previous seminal paper, we reported experimental observation by high-angle annular dark field-scanning transmission electron microscope (HAADF-STEM) images [12] of single GaSe layer with a structure different from the one reported so far exists near the $\mathrm{GaSe}(0001) / \mathrm{Ge}(111)$ interface. In this structure, $\mathrm{Se}$ atoms are coordinated in a trigonal-antiprismatic way with respect to the Ga dimer as shown in Fig. 1(b). We name this GaSe crystal with this structure the "antiprismatic (AP) phase." Monolayer AP-phase GaSe has a centrosymmetric crystal structure in contrast to the noncentrosymmetric crystal structure of monolayer P-phase GaSe.

The structural relationship between the P-phase and APphase GaSe monolayers is reminiscent of that between trigonal-prismatic and octahedral structures in transitionmetal dichalcogenides (TMDCs) which are also LMCs. Depending on conditions such as alkali-metal intercalation, both structures can be stabilized, which have very different properties [14-16]. For example, trigonal-prismatic $\mathrm{MoS}_{2}$ is semiconducting, while octahedral $\mathrm{MoS}_{2}$ is metallic [17,18]. On the other hand, for group-III metal monochalcogenides such as GaSe, the variation in intralayer structure has hardly been discussed. So far, only one theoretical report presented the results of calculations on monolayer indium chalcogenides with antiprismatic structure named $\beta$-InX [19].

In this paper, we report the structural stability and electronic states of AP-phase GaSe monolayer obtained by 
(a)

(b)
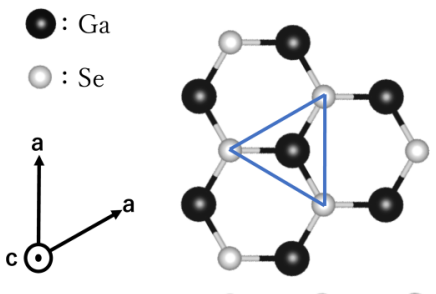

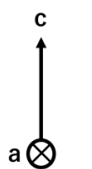
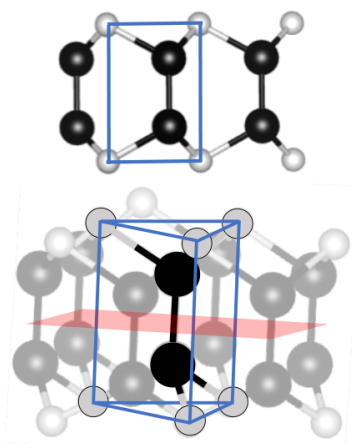
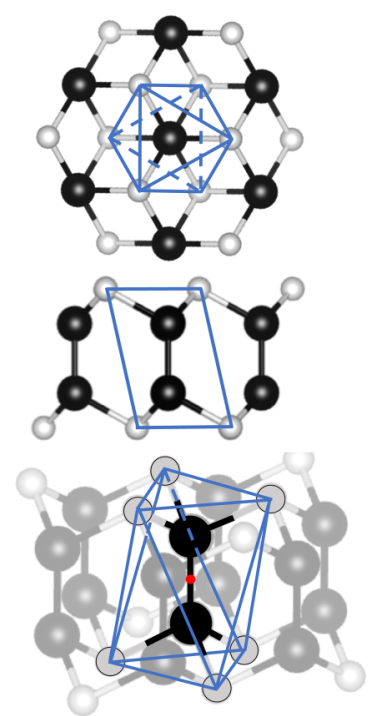

FIG. 1. Top view (top), side view (middle), and perspective view (bottom) of crystal structures of (a) prismatic (P)-phase and (b) antiprismatic (AP)-phase GaSe monolayers. Blue thin lines highlight (a) the triangular prism and (b) antiprism. P phase has mirror symmetry [mirror plane in red in the perspective view of (a)] and no inversion symmetry, while AP phase has inversion symmetry [inversion center indicated as red point in the perspective view of (b)]. Images of structures were produced with VESTA [47].

first-principles calculations based on the density functional theory (DFT). Comparing the results of calculations carried out for P phase, we discuss the relative stability of the two phases, and the possible formation mechanism of the newly found AP-phase GaSe.

\section{COMPUTATIONAL DETAILS}

DFT calculations have been performed using the OpenMX code [20-23]. This code is based on norm-conserving pseudopotentials and optimized pseudoatomic localized basis functions [24]. The exchange-correlation functional was treated within generalized gradient approximation by Perdew, Burke, and Ernzerhof (GGA-PBE) [25,26]. The basis functions Ga7.0-s3p3d3f1 and Se7.0-s3p3d2f1 are used in the calculations, which were generated by the confinement scheme, and optimized by a variational optimization method [20]. The pseudopotentials of $\mathrm{Ga}$ and Se atoms include 13 and 6 valence electrons, respectively. The accuracy of the basis functions and pseudopotentials we used were carefully benchmarked by the delta gauge method [27]. In the calculations for band structures, the effect of SOC was incorporated through the $j$-dependent pseudopotentials [28]. The regular mesh of 300 Ry in real space was used for the numerical integrations and for the solution of the Poisson equation. A $(7 \times 7 \times 1) k$-point mesh was used to discretize the first Brillouin zone in this study. The density of states (DOS) has been calculated with a tetrahedron method on a $(12 \times 12 \times 1) k$-point mesh. All atomic positions have been relaxed until the residual

force on each atom has reached values of less than 0.0003 hartree/bohr. The vacuum space along the $c$ direction is taken to be more than $15 \AA$ to avoid the spurious interaction between slabs.

In order to assure structural stability of AP-phase GaSe, we have performed $a b$ initio molecular dynamics (MD) calculations. The $(3 \times 3)$ supercells at equilibrium in-plane lattice constants of P- and AP-phase GaSe were used for the MD calculations. We used a 3 -fs time step and set the temperature to $773 \mathrm{~K}$ with the Nosé-Hoover thermostat [29-31]. The substrate temperature used to grow GaSe thin films was chosen as the set temperature [12]. The calculations were performed for a total of 500 time steps (1.5 ps). To analyze the energy barrier between P- and AP-phase GaSe, 16 transition images are adopted using the nudged elastic band (NEB) method where the minimum-energy path between the two configurations at equilibrium lattice constant of P-phase GaSe can be found [32]. Here, the maximum force is set to be less than 0.0003 hartree/bohr.

\section{RESULTS AND DISCUSSION}

\section{A. Structural stability}

Since AP-phase GaSe monolayer was experimentally observed only in the vicinity of the film-substrate interface of epitaxial $\mathrm{GaSe}(0001)$ thin films grown on $\mathrm{Ge}(111)$ substrates [12], the effect of in-plane strain on the structural stability was studied by calculating total energies at different in-plane lattice constants. The calculated total energies per chemical formula unit versus in-plane lattice constants of P-phase and AP-phase GaSe monolayers are plotted in Fig. 2. The lattice constants resulting in the lowest energies for P-phase and AP-phase GaSe monolayers were determined to be 3.81 and $3.82 \AA$, respectively. Note that the experimentally obtained lattice constant of bulk $\varepsilon$-GaSe crystal is $3.74 \AA$ [33]. With these stable lattice constants, the $\mathrm{P}$ phase is more stable

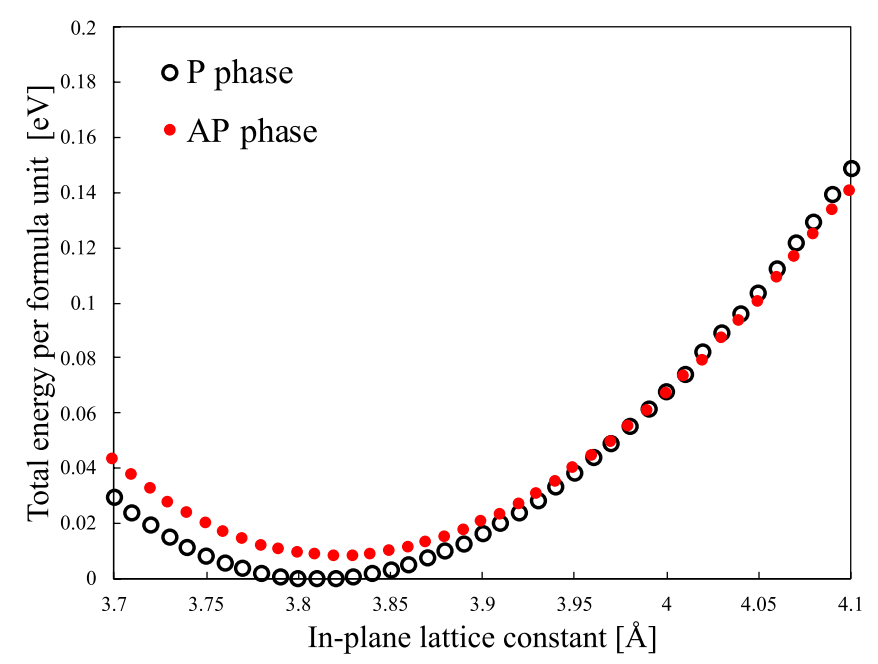

FIG. 2. Energy vs lattice constant curves for P- and AP-phase monolayer GaSe. The lattice constants of the structurally optimized P-phase and AP-phase GaSe monolayers are 3.81 and $3.82 \AA$, respectively. 
compared to the AP phase. Note, however, that the energy difference between the two phases at the lattice constants of $3.81 \AA$ is approximately $8 \mathrm{meV}$ per formula unit. This energy difference is about the same as the cohesive energy difference (13 meV per unit cell), calculated for $\alpha$-(P-phase) and $\beta$-(AP-phase) InX monolayers [19]. It is well known that wurtzite (WZ) and zinc-blende (ZB) structures of GaN coexist in an epifilm grown by molecular beam epitaxy (MBE) [34]. The coordination of $\mathrm{WZ}$ and $\mathrm{ZB}$ structures are similar to the P-phase and AP-phase GaSe, respectively. ZB-GaN is known as a metastable phase, and the energy difference between $\mathrm{WZ}$ and $\mathrm{ZB}$ phases was reported to be in the range of 8 meV/f.u. [35] to $30 \mathrm{meV} / \mathrm{f} . u$. [36]. The experimental studies revealed that necessary growth conditions for $\mathrm{ZB}-\mathrm{GaN}$ is low substrate temperature [37], which indicates that the nonequilibrium condition is important for the nucleation and growth of metastable ZB phase. The calculated energy difference between the P and AP phases of GaSe is similar to or less than that between two polymorphs of GaN. This is consistent with our previous experimental observation of P-phase and AP-phase GaSe coexisting in thin films grown by MBE which is known as a nonequilibrium process [12].

In order to investigate if these two phases of monolayer GaSe can transform into each other, MD calculations and NEB calculations have been performed. The MD calculation results, performed at each equilibrium in-plane lattice constant, indicate that there is no structural phase transition between $\mathrm{P}$ and $\mathrm{AP}$ at $773 \mathrm{~K}$, because P- and AP-phase GaSe oscillate thermally around their respective structures. The energy barrier from $\mathrm{P}$ phase to AP phase at in-plane lattice constant of $3.81 \AA$ has been confirmed by the NEB calculations to be about $1.1 \mathrm{eV}$ per formula unit. The total energy along the minimum-energy path and selected images of transition states are plotted in Fig. 3. The large energy barrier of $1.1 \mathrm{eV}$ exists for the phase transition because the Ga-Se bonds need to be broken (see the evolution of crystal structure in the inset of Fig. 3). AP-phase GaSe is most likely not formed by phase transition from the already nucleated $\mathrm{P}$ phase, but rather by direct nucleation of the AP phase. The above results suggest that AP-phase GaSe can be grown by nonequilibrium processes, such as low-temperature MBE.

Furthermore, the energy difference between trigonalprismatic and octahedral structures in TMDCs (e.g., $\mathrm{MoS}_{2}$, $\mathrm{MoTe}_{2}$, and $\mathrm{WS}_{2}$ ) is about $0.5-0.9$ eV/f.u. [38] which is about 100 times larger than that of GaSe or $\beta$-InX. It is assumed that the structural stability between the two structures is determined by the balance between the chalcogen-metal bond and the ion repulsion between the chalcogens [39,40]. Since the distance between the chalcogen atoms in the monolayers of III-VI layered materials are larger than that for TMDCs, the interaction between chalcogen atoms should be small. Therefore, the small calculated energy difference between the two structures of GaSe compared to those for TMDCs is consistent with the earlier studies $[39,40]$.

Figure 2 shows that the energy difference between the two phases decreases by increasing the lattice constants, and the relative stability reverses at the lattice constant of $3.96 \AA$. The result tells us that, although the energy difference of the two

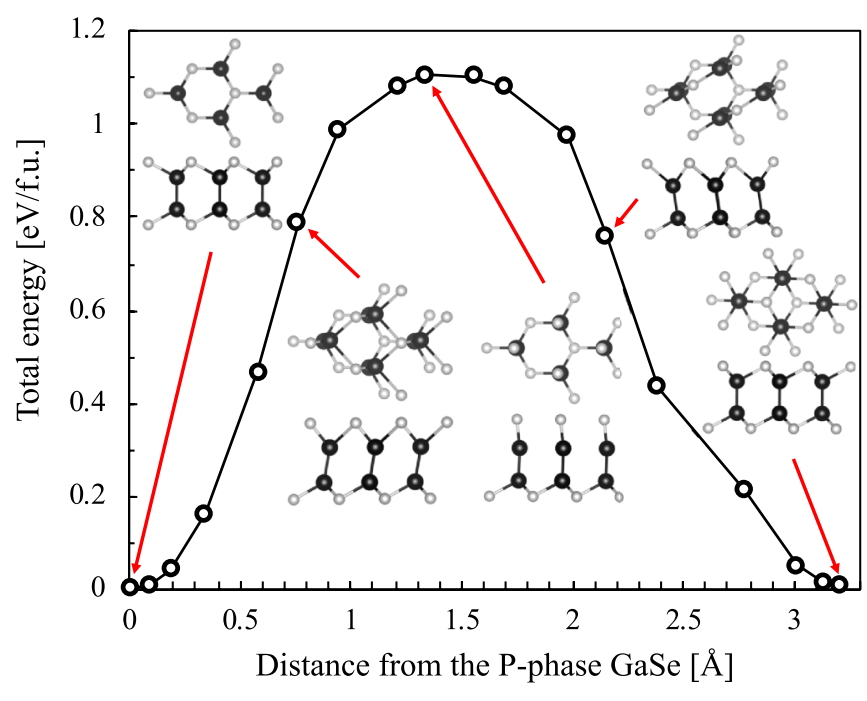

FIG. 3. The transition of relative total energy per formula unit from P-phase (left) to AP-phase (right) monolayer GaSe by the NEB calculation at in-plane lattice constant of $3.81 \AA$. The energy barrier between both phases is $1.1 \mathrm{eV}$ per formula unit. The top and side views of GaSe structure at selected distance from P-phase GaSe are presented. The energy difference between P-phase and AP-phase $\mathrm{GaSe}$ is $8 \mathrm{meV}$ per formula unit.

phases is very small, the $\mathrm{P}$ phase is more stable than the AP phase at equilibrium lattice constants, which is consistent with the experimental observations [12]. On the other hand, the AP phase tends to become more stable than the $P$ phase as the inplane lattice constant increases. In other words, the calculation result suggests that the AP phase could be stabilized by the in-plane tensile strain.

In our previous study, the AP-phase GaSe layer was observed at the first or the second layer from the Ge(111) substrate surface in HAADF-STEM images [12]. The optimized lattice parameter of in-plane $\mathrm{Ge}(111)$ surface calculated for bulk under the same condition was $4.09 \AA$, which is $7 \%$ larger than the optimized lattice constant of monolayer GaSe. At the $\mathrm{GaSe}(0001) / \mathrm{Ge}(111)$ interface, most of the strain resulting from the difference between the lattice constants of half-layer GaSe terminated Ge substrate and GaSe thin film is considered to be mitigated by the van der Waals interaction. However, our experimental observation and calculation results suggest that there could be a tensile environment at the vicinity of the film-substrate interface even in the van der Waals epitaxy growth.

To reveal the origin of the difference in structural stability between P- and AP-phase GaSe, we analyzed their optimized structures. The in-plane lattice constant dependence of layer thicknesses and the bond lengths of Ga-Ga and Ga-Se in Pand AP-phase GaSe are shown in Figs. 4(a)-4(c). The layer thickness is defined as the difference between the $c$-axis coordinate of Se atoms. The obtained structural parameters and energy difference at selected lattice constants are summarized in Table I. As the in-plane lattice constant increases, the layer thickness becomes smaller in both phases. Note that at the same in-plane lattice constant, the layer thickness of the AP phase is always smaller than that of the $\mathrm{P}$ phase. This is 

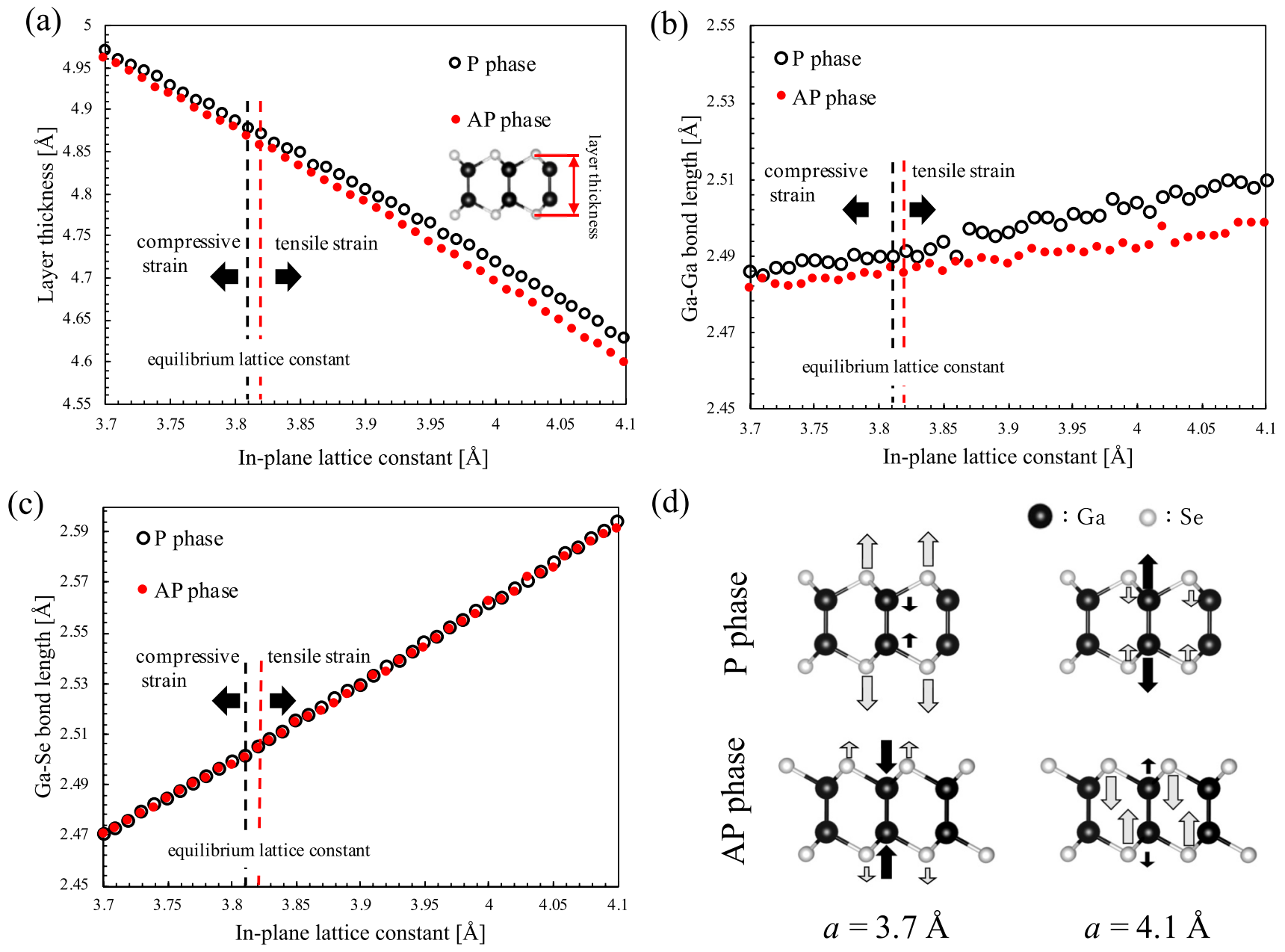

(d)

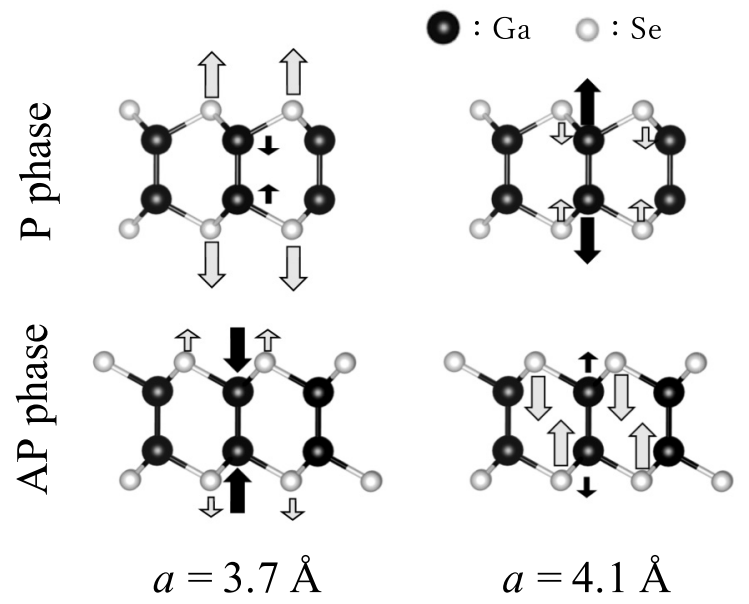

FIG. 4. Structural parameters as a function of the in-plane lattice constant for the P- and AP-phase monolayer GaSe. (a) Layer thickness, (b) Ga-Ga, and (c) Ga-Se bond. Layer thickness is the difference between the $c$ coordinates of Se atoms shown in the inset of (a). Black and red dashed lines are equilibrium in-plane lattice constants of P- and AP-phase GaSe, respectively. (d) Magnitude of displacement from the structure at the equilibrium in-plane lattice constant. Black and gray color indicate Ga and Se atoms, respectively, and the length of the arrows indicate the magnitude of displacements.

considered to be due to the difference in Se-Se repulsion. In the $\mathrm{P}$ phase, where two $\mathrm{Se}$ atoms at the outermost layers sit in the same in-plane position, stronger Se-Se repulsion can be expected compared to the AP phase, where the in-plane positions of Se atoms between the outermost layers are different. This explanation is also supported by the calculation results showing that the difference in layer thickness between the two phases increases with the increase of the in-plane lattice constant.

As for the bond lengths, there is no difference in the inplane lattice constant dependence of the Ga-Se bond length between the two phases, while there is a slight difference in the case of Ga-Ga bond length. The Ga-Ga bond length tends to increase as the in-plane lattice constant increases. The

TABLE I. The calculated structural parameters and energy difference with respect to AP-phase GaSe. Layer thickness is the difference between the $c$ coordinates of Se atoms shown in Fig. 4(a).

\begin{tabular}{|c|c|c|c|c|c|c|c|}
\hline \multirow{2}{*}{$\begin{array}{l}\text { In-plane lattice } \\
\text { constant }(\AA)\end{array}$} & \multicolumn{2}{|c|}{ Layer thickness $(\AA)$} & \multicolumn{2}{|c|}{ Ga-Se bond length $(\AA)$} & \multicolumn{2}{|c|}{ Ga-Ga bond length $(\AA)$} & \multirow{2}{*}{$\begin{array}{c}\Delta E^{\mathrm{P}-\mathrm{AP}} \\
\text { (meV/f.u.) }\end{array}$} \\
\hline & $\mathrm{P}$ & $\mathrm{AP}$ & $\mathrm{P}$ & AP & $\mathrm{P}$ & AP & \\
\hline 3.81 & 4.88 & 4.87 & 2.50 & 2.50 & 2.49 & 2.49 & -8 \\
\hline 4.10 & 4.63 & 4.60 & 2.59 & 2.59 & 2.51 & 2.50 & 8 \\
\hline
\end{tabular}



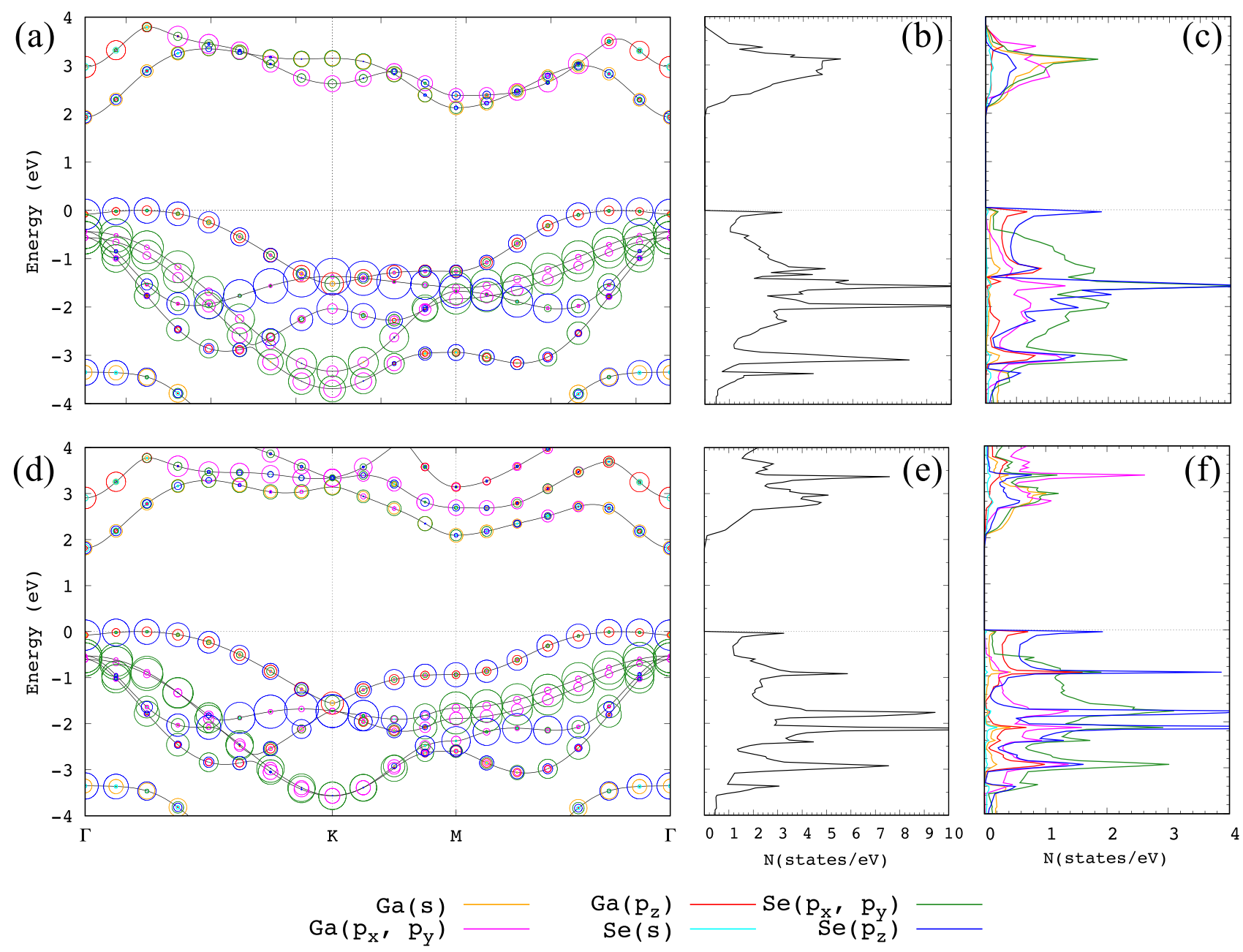

FIG. 5. (a)-(c) Band structures, DOS, and PDOS for the P-phase GaSe monolayer with the equilibrium in-plane lattice constant. (d)-(f) Same for AP-phase GaSe monolayer at its equilibrium in-plane lattice constant. All were calculated without including SOC. Pseudoatomic orbital contribution is depicted on the band structures by the color and size of the circles.

Ga-Ga bond length of the AP phase is always smaller than that of the $\mathrm{P}$ phase for the same lattice constant.

Together with the $\mathrm{P}$ phase, the changes in layer thickness and $\mathrm{Ga}-\mathrm{Ga}$ bond lengths of AP-phase GaSe under compressive $(a=3.7 \AA)$ and tensile $(a=4.1 \AA)$ strains are depicted in Fig. 4(d). Under tensile condition, Se atoms are shifted inward, in the direction perpendicular to the layer, in both phases, however, the magnitude of the shift is larger in the AP phase compared to the $\mathrm{P}$ phase, probably reflecting the difference in Se-Se repulsive forces between the two phases. While the Ga-Se bond lengths are similar in both phases [which is shown in Fig. 4(c)], for the $\mathrm{P}$ phase, in which the inward shift of Se is small, the increase in Ga-Ga bond length becomes significant. On the other hand, the increased Ga-Ga bond length in the AP phase becomes almost similar to that of the $\mathrm{P}$ phase at the equilibrium lattice constant. These calculation results suggest that the increase in $\mathrm{Ga}-\mathrm{Ga}$ bond lengths under tensile strain changes the sign of the difference of energy between the P and AP phases. Note that the same stabilizing mechanism of the AP phase can be suggested from the calcu- lation results for gallium sulfide (GaS) (see the Supplemental Material [41]).

\section{B. Band structure}

Electronic band structures for P-phase and AP-phase GaSe monolayers have been calculated for their optimized structures with lattice constants giving lowest energies. The resulting dispersion relations for the two phases, plotted along the high-symmetry directions of the two-dimensional hexagonal Brillouin zone $(\Gamma-K-M-\Gamma)$, are shown in Figs. 5(a) and 5(d) without SOC. The zero energy is adjusted to the top of the valence band.

Both phases are indirect-gap semiconductors, primarily due to the valence-band maximum lying between the $\Gamma$ and $K$ points. The indirect gaps of $\mathrm{P}$ and $\mathrm{AP}$ phases are 1.94 and $1.83 \mathrm{eV}$, respectively. Kohn-Sham eigenvalues of valence-band maximum (VBM) and conduction-band minimum (CBM) at $\Gamma$ point are summarized in Table II. The difference in the band gap between $\mathrm{P}$ and AP phases is about $5 \%$, whether SOC is taken into account or not. Experimental 
TABLE II. The valence-band maximum (VBM), conduction-band minimum (CBM) at $\Gamma$ point, and indirect band gap from calculated electronic structure of P- and AP-phase monolayer GaSe with/without SOC at their respective equilibrium lattice constants.

\begin{tabular}{lccc}
\hline \hline & \multicolumn{2}{c}{ P phase $(a=3.81 \AA)$} & AP phase $(a=3.82 \AA)$ \\
\cline { 2 - 4 } & w/o SOC & with SOC & w/o SOC \\
\hline VBM $(\mathrm{eV})$ & -0.079 & -0.053 & -0.074 \\
$\mathrm{CBM}(\mathrm{eV})$ & 1.93 & 1.90 & 1.81 \\
Indirect band gap $(\mathrm{eV})$ & 1.93 & 1.90 & 1.81 \\
\hline \hline
\end{tabular}

band-gap values of P-phase monolayer GaSe measured by STS and CL are $3.5 \pm 0.05 \mathrm{eV}$ [7] and $3.3 \mathrm{eV}$ [8], respectively.

The valence band of the AP-phase GaSe is similar to that of P-phase GaSe near the $\Gamma$ point. However, some differences arise at the $K$ point, where a doubly degenerate band appears at the second and third highest valence bands and second lowest conduction band. In addition, the VBM at the $M$ point has an energy about $0.3 \mathrm{eV}$ higher in the AP phase than in the $\mathrm{P}$ phase. Since both bands are mainly composed of Se $p_{z}$, the difference in band structure can be explained by the breaking of mirror symmetry.
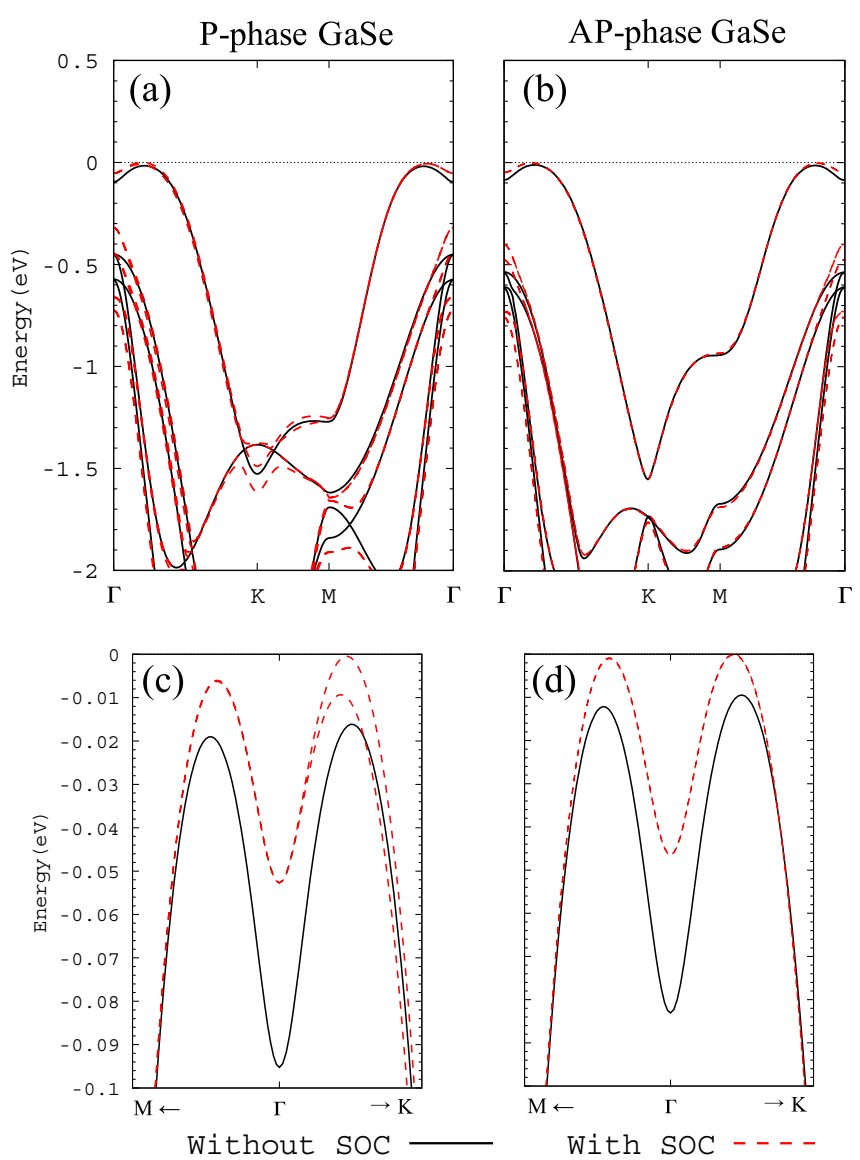

FIG. 6. Calculated band structures of P- and AP-phase monolayer GaSe at their respective equilibrium lattice constants. Bands near topmost valence band are shown, which are calculated without (solid line) and with (dashed line) SOC. Band structures (a), (b) along high-symmetry directions, and (c), (d) around $\Gamma$ point, respectively.
The density of states (DOS) and projected density of state (PDOS) of P-phase and AP-phase GaSe monolayers are shown in Figs. 5(b), 5(c), 5(e), and 5(f), respectively. The DOS of the AP phase in the valence band is similar to that of P-phase GaSe. Both results show a sharp Van Hove singularity at the VBM, similar to those which have been discussed in monolayer P-phase GaSe, GaS, and InSe, originating from the ring-shaped band extremum [43].

Band structures near the topmost valence band with and without SOC of both phases are shown in Fig. 6. In contrast to the band structures of bulk GaSe crystals, the VBM has a local minimum at the $\Gamma$ point in monolayer GaSe which is sometimes called "sombrero" dispersion [43]. As shown in Figs. 6(c) and 6(d), while valence bands around the $\Gamma$ point calculated without SOC are similar for both phases, those calculated with SOC show a striking difference. Band splitting originating from SOC appeared along the $\Gamma-K$ direction in the P phase [Fig. 6(c)] [10], but the band splitting in the AP phase is negligibly small [Fig. 6(d)]. Although ferromagnetism and half-metallicity are expected to emerge in monolayer P-phase GaSe with a spin-orbit split band [10], further study is necessary to find out whether the same behavior can be expected for monolayer AP-phase GaSe.

\section{CONCLUSIONS}

Through first-principles calculations, we found that the AP-phase GaSe monolayer is metastable with a very small energy difference to the conventional P-phase GaSe, and this relative stability can be reversed under in-plane tensile strain. This result is consistent with the experimental observation that AP-phase GaSe monolayer was observed only near the $\mathrm{Ge}(111)$ substrate. The band structures of both phases were similar, but in the case of AP-phase GaSe, the bands with strong Se orbital character degenerates at the $K$ point. The indirect band gap of AP-phase GaSe is about $0.1 \mathrm{eV}$ smaller than that of P-phase GaSe.

This unconventional GaSe phase may have been formed in previous growth experiments, especially in nonequilibrium processes. Actually, AP-phase-like GaSe can be seen in published STEM images, but is not discussed at all [44]. Since AP-phase GaSe has centrosymmetricity, this monolayer may have no second-order nonlinear optical properties such as second-harmonic generation (SHG). The experimental observation of zero SHG signal in $\beta$-GaSe which has centrosymmetricity due to stacking supports the above prediction [45]. Since the optical-absorption selection rules of P-phase GaSe is closely related to the mirror symmetry of the polymorph, that of the AP phase is of interest [46]. This contributes to a better 
understanding of the experimental results of optical properties, considering the coexistence of AP-phase GaSe in GaSe thin films made by MBE or other nonequilibrium processes.

\section{ACKNOWLEDGMENTS}

This work was supported by Shibuya Science Culture and Sports Foundation. A part of this work was carried out using the facilities in JAIST, supported by Nanotechnology Platform Program (Molecule and Material Synthesis) of the Ministry of Education, Culture, Sports, Science and Technology (MEXT), Japan. H.N. and A.F. acknowledge support from the joint research program of the Institute for Solid State Physics, University of Tokyo. A.F. acknowledges supports from JSPS KAKENHI Grant No. JP19K05204.
[1] M. Xu, T. Liang, M. Shi, and H. Chen, Chem. Rev. 113, 3766 (2013).

[2] S. Z. Butler, S. M. Hollen, L. Cao, Y. Cui, J. A. Gupta, H. R. Gutiérrez, T. F. Heinz, S. S. Hong, J. Huang, A. F. Ismach et al., ACS Nano 7, 2898 (2013).

[3] N. C. Fernelius, Prog. Cryst. Growth Charact. Mater. 28, 275 (1994).

[4] R. Clasen, G. Harbeke, A. Krost, F. Lévy, O. Madelung, K. Maschke, G. Nimtz, B. Schlicht, F. J. Schmitte, and J. Treusch, Landolt-Bornstein, in Semiconductors Subvolume f: Physics of Non-Tetrahedrally Bonded Binary Compounds II, edited by $\mathrm{O}$. Madelung (Springer-Verlag, Berlin, 1983), pp. 18-19.

[5] T. J. Bastow, I. D. Campbell, and H. J. Whitfield, Solid State Commun. 39, 307 (1981).

[6] X. Li, M.-W. Lin, A. A. Puretzky, J. C. Idrobo, C. Ma, M. Chi, M. Yoon, C. M. Rouleau, I. I. Kravchenko, D. B. Geohegan, and K. Xiao, Sci. Rep. 4, 5497 (2015).

[7] Z. Ben Aziza, D. Pierucci, H. Henck, M. G. Silly, C. David, M. Yoon, F. Sirotti, K. Xiao, M. Eddrief, J.-C. Girard, and A. Ouerghi, Phys. Rev. B 96, 035407 (2017).

[8] C. S. Jung, F. Shojaei, K. Park, J. Y. Oh, H. S. Im, D. M. Jang, J. Park, and H. S. Kang, ACS Nano 9, 9585 (2015).

[9] Z. Zhu, Y. Cheng, and U. Schwingenschlögl, Phys. Rev. Lett. 108, 266805 (2012).

[10] T. Cao, Z. Li, and S. G. Louie, Phys. Rev. Lett. 114, 236602 (2015).

[11] S. Takasuna, J. Shiogai, S. Matsuzaka, M. Kohda, Y. Oyama, and J. Nitta, Phys. Rev. B 96, 161303(R) (2017).

[12] T. Yonezawa, T. Murakami, K. Higashimine, A. Fleurence, Y. Oshima, and Y. Yamada-Takamura, Surf. Interface Anal. 51, 95 (2019).

[13] A. Koma, Thin Solid Films 216, 72 (1992).

[14] G. Eda, T. Fujita, H. Yamaguchi, D. Voiry, M. Chen, and M. Chhowalla, ACS Nano 6, 7311 (2012).

[15] M. Chhowalla, H. S. Shin, G. Eda, L.-J. Li, K. P. Loh, and H. Zhang, Nat. Chem. 5, 263 (2013).

[16] Y. Cheng, A. Nie, Q. Zhang, L.-Y. Gan, R. Shahbazian-Yassar, and U. Schwingenschlogl, ACS Nano 8, 11447 (2014).

[17] G. Eda, H. Yamaguchi, D. Voiry, T. Fujita, M. Chen, and M. Chhowalla, Nano Lett. 11, 5111 (2011).

[18] M. Calandra, Phys. Rev. B 88, 245428 (2013).

[19] V. Zólyomi, N. D. Drummond, and V. I. Fal'ko, Phys. Rev. B 89, 205416 (2014).

[20] T. Ozaki, Phys. Rev. B 67, 155108 (2003).

[21] T. Ozaki, http://www.openmx-square.org/.

[22] T. Ozaki and H. Kino, Phys. Rev. B 72, 045121 (2005).
[23] T. Ozaki and H. Kino, Phys. Rev. B 69, 195113 (2004).

[24] I. Morrison, D. M. Bylander, and L. Kleinman, Phys. Rev. B 47, 6728 (1993).

[25] W. Kohn and L. J. Sham, Phys. Rev. 140, A1133 (1965).

[26] J. P. Perdew, K. Burke, and M. Ernzerhof, Phys. Rev. Lett. 77, 3865 (1996).

[27] K. Lejaeghere, G. Bihlmayer, T. Bjorkman, P. Blaha, S. Blugel, V. Blum, D. Caliste, I. E. Castelli, S. J. Clark, A. Dal Corso et al., Science 351, aad3000 (2016).

[28] G. Theurich and N. A. Hill, Phys. Rev. B 64, 073106 (2001).

[29] S. Nosé, J. Chem. Phys. 81, 511 (1984).

[30] S. Nosé, Mol. Phys. 52, 255 (1984).

[31] W. G. Hoover, Phys. Rev. A 31, 1695 (1985).

[32] G. Henkelman, B. P. Uberuaga, and H. Jónsson, J. Chem. Phys. 113, 9901 (2000).

[33] J. C. J. M. Terhell, V. A. M. Brabers, and G. E. van Egmond, J. Solid State Chem. 41, 97 (1982).

[34] Y. Zhao, C. W. Tu, I.-T. Bae, and T.-Y. Seong, Appl. Phys. Lett. 74, 3182 (1999).

[35] T. Ito and Y. Kangawa, J. Cryst. Growth 235, 149 (2002).

[36] C.-Y. Yeh, Z. W. Lu, S. Froyen, and A. Zunger, Phys. Rev. B 46, 10086 (1992).

[37] B. M. Shi, M. H. Xie, H. S. Wu, N. Wang, and S. Y. Tong, Appl. Phys. Lett. 89, 151921 (2006).

[38] K.-A. N. Duerloo, Y. Li, and E. J. Reed, Nat. Commun. 5, 4214 (2014).

[39] R. Huisman, R. de Jonge, C. Haas, and F. Jellinek, J. Solid State Chem. 3, 56 (1971).

[40] N. Kuroda and Y. Nishina, Kagaku Sosetsu 42, 202 (1983) (published in Japanese).

[41] See Supplemental Material at http://link.aps.org/supplemental/ 10.1103/PhysRevB.102.235407 for the GaS case, which includes Refs. [42] and [47].

[42] H. d'Amour and W. B. Holzapfel, Solid State Commun. 44, 853 (1982).

[43] D. V. Rybkovskiy, A. V. Osadchy, and E. D. Obraztsova, Phys. Rev. B 90, 235302 (2014).

[44] C. H. Lee, S. Krishnamoorthy, D. J. O’Hara, M. R. Brenner, J. M. Johnson, J. S. Jamison, R. C. Myers, R. K. Kawakami, J. Hwang, and S. Rajan, J. Appl. Phys. 121, 094302 (2017).

[45] X. Zhou, J. Cheng, Y. Zhou, T. Cao, H. Hong, Z. Liao, S. Wu, H. Peng, K. Liu, and D. Yu, J. Am. Chem. Soc. 137, 7994 (2015).

[46] G. Antonius, D. Y. Qiu, and S. G. Louie, Nano Lett. 18, 1925 (2018).

[47] K. Momma and F. Izumi, J. Appl. Cryst. 44, 1272 (2011). 